Biochimica et Biophysica Acta, 557 (1979) 1-8

(C) Elsevier/North-Holland Biomedical Press

BBA 78509

\title{
INTERACTIONS OF NEOMYCIN WITH MONOMOLECULAR FILMS OF POL YPHOSPHOINOSITIDES AND OTHER LIPIDS
}

\author{
SHAHID LODHI, NORMAN D. WEINER and JOCHEN SCHACHT * \\ College of Pharmacy, Kresge Hearing Research Institute and Department of Biological \\ Chemistry, The University of Michigan, Ann Arbor, MI 48109 (U.S.A.)
}

(Received February 21st, 1979)

Key words: Neomycin; Polyphosphoinositide; Lipid-antibiotic interaction

\section{Summary}

The interactions of calcium and the aminoglycosidic antibiotic, neomycin, with various lipids were investigated in monomolecular films. Lipids were spread over a subphase of $0.05 \mathrm{M} \mathrm{N}$-2-hydroxyethylpiperazine- $N^{\prime}$-2-ethanesulfonic acid, $\mathrm{pH} 7.0$, and $\mathrm{NaCl}$ to give an ionic strength of 0.2 . Measurements of surface pressure $(\pi)$ were taken with a Wilhelmy balance. In the absence of $\mathrm{Ca}^{2+}, 1 \mu \mathrm{M}-1 \mathrm{mM}$ neomycin in the subphase decreased $\pi$ (i.e. condensed films) of all acidic lipids tested. In the presence of $1 \mathrm{mM} \mathrm{Ca}^{2+}$, neomycin did not change $\pi$ of films of phosphatidylserine, phosphatidylinositol and phosphatidic acid while it lowered $\pi$ of cardiolipin and cerebroside sulfate films. A unique pattern of interaction was observed with polyphosphoinositide monolayers. In the absence of $\mathrm{Ca}^{2+}, 1 \mu \mathrm{M}$ neomycin decreased $\pi$ followed by an increase of $\pi$ at higher neomycin concentrations. $\mathrm{Ca}^{2+}(1 \mathrm{mM})$ condensed the film significantly more than did neomycin. However, as little as $1 \mu \mathrm{M}$ neomycin induced expansion of the calcium/lipid film which at $1 \mathrm{mM}$ neomycin reached the same $\pi$ as in the absence of $\mathrm{Ca}^{2+}$. Such expansion was observed at all pressures of the film including the collapse pressure indicating a strong 'complex' between the drug and polyphosphoinositide not antagonized by $\mathrm{Ca}^{2+}$. In the absence of possible hydrophobic interactions, both the condensation and the expansion of the film should be mediated by ionic forces. Combined in vivo and in vitro evidence is discussed to suggest the polyphosphoinositides as the physiological receptors for aminoglycosides in the mammalian cell membrane.

* To whom correspondence should be addressed.

Abbreviation: Hepes, $N$-2-hydroxyethylpiperazine- $N$ '-2-ethanesulfonic acid. 


\section{Introduction}

It is well-established that the first step of the action of many hormones and drugs is their binding to receptor sites in the cell membrane. Receptor sites have been characterized as proteins in the case of many neurotransmitters and hormones, as glycoproteins in case of lectins, glycolipids in the case of glycoprotein hormones and bacterial toxins [1,2]. In all these cases the observation has been that the recognition of the cell receptor by the agonist is highly specific. In contrast, drug actions involving phospholipids do not seem to exhibit such a specificity. Local anesthetics, for instance, which form complexes with phospholipids based on hydrophobic and ionic interactions [3], or basic toxins such as the antibiotic polymyxin $B$, which require acidic phospholipids for their action, have no apparent preference for a specific lipid [4].

We have previously reported in vivo and in vitro actions of neomycin and related aminoglycosidic antibiotics on the metabolism of polyphosphoinositides, phosphatidylinositol phosphate and phosphatidylinositol bisphosphate * in mammalian tissues [5-7]. The action of this drug appeared to be directed specifically against these lipids and was attributed to a direct binding of neomycin to the polyphosphoinositides [8]. Preliminary experiments with monomolecular films confirmed interactions between the antibiotic and these lipids $[9]$.

The objective of this study was to investigate whether there is a specific interaction of neomycin with polyphosphoinositides as compared to other anionic lipids as suggested by the in vivo results **.

\section{Materials and Methods}

Reagents. Phospholipids were obtained from Sedary Research Laboratories, London, Ontario. Cerebroside sulfate was a gift from Dr. N. Radin, University of Michigan. Polyphosphoinositides were prepared by chromatography on immobilized neomycin [10]. Neomycin sulfate was USP Grade. All water was triple distilled from an all-glass still.

Surface pressure vs. surface area ( $\pi$ vs. A) studies (compression isotherms). The film balance assembly has been described previously [11]. Briefly, it consisted of a rectangular Teflon trough with a movable Teflon barrier. A Wilhelmy balance with a thin platinum plate, roughened to ensure complete wetting, was used to record surface pressures as a function of surface area. In order to verify that no surface-active agents were present in the aqueous solutions and organic solvents the surface tension was recorded at various trough areas at the beginning of each experiment. A clean surface was indicated by the same surface tension at all positions of the barrier. The buffer in the aqueous subphase was $50 \mathrm{mM}$ sodium Hepes, $\mathrm{pH} 7.0$, and its ionic strength was adjusted to 0.2 with sodium chloride heated for $12 \mathrm{~h}$ at $700^{\circ} \mathrm{C}$. Lipids were spread on

\footnotetext{
* 1-(3-sn-phosphatidy1)-D-myoinositol-4-phosphate (1-phosphatidylinositol-4-phosphate) and 1-(3-snphosphatidyl)-n-my oinositol-4,5-bisphosphate (1-phosphatidylinositol-4,5-bisphosphate).

** Presented in part at the symposium on 'Cyclitols and Phosphoinositides', held in East Lansing, MI. U.S.A., June 1977 .
} 
the subphase as solutions in $n$-hexane/ethanol (95:5, v/v), except polyphosphoinositides. These were a mixture of phosphatidylinositol phosphate and phosphatidylinositol bisphosphate in an approximate $1: 2$ molar ratio and were spread as a solution in $n$-hexane/ethanol/chloroform ( $80: 5: 15$, by vol.). Approximately $0.2 \mu \mathrm{g}$ lipid $/ \mathrm{cm}^{2}$ was required to obtain the initial surface pressure. After spreading, the solvent was allowed to evaporate and the film to stabilize for 15 min. Measurements were made 2 min after compressing the film to a new surface area. It had previously been determined that stable pressures were obtained after this time. For each curve of surface pressure vs. surface area at least two readings each were taken at 26-30 different trough areas. Only duplicate readings agreeing to within 0.1 dyne $/ \mathrm{cm}$ were acceptable. For the sake of clarity the averages of these values are plotted as smooth curves. All experiments were performed at $25 \pm 3^{\circ} \mathrm{C}$.

Constant area studies. A Teflon beaker with an inner diameter of $8 \mathrm{~cm}$ and a Wilhelmy balance were used to measure surface pressure. A stationary $3 \mathrm{ml}$ syringe whose needle remained immersed into the subphase (volume $100 \mathrm{ml}$ ) throughout the experiment delivered additions to the buffer beneath the film. A Teflon-coated magnetic bar stirred the solutions at slow speed without disturbing the film. The amount of lipid spread over the buffer varied from 0.2 to $0.5 \mu \mathrm{g} / \mathrm{cm}^{2}$ depending on the desired initial surface pressures which were recorded after the film had stabilized for $10 \mathrm{~min}$. Neomycin was then injected into the subphase with the final concentration of the drug varying from $10^{-8}$ to $10^{-3} \mathrm{M}$. After each addition of neomycin the subphase was mixed for 15 min. When calcium was present in the experiments it was added to the buffer before the lipid film was formed.

Two readings each were taken at ten different drug concentrations over the range indicated above. Experiments were acceptable only if duplicate readings agreed within $0.1 \mathrm{dyne} / \mathrm{cm}$ and the curves resulting from the average values are shown.

\section{Results and Discussion}

\section{Compression isotherms}

Compression isotherms are a conventional technique to characterize lipid films and their interactions with drugs or ions. The addition of $1 \mathrm{mM}$ calcium to a phosphatidylserine film (Fig. 1A) shifted the $\pi$ vs. $A$ curve to the left at all surface areas, indicating a condensation of the film. Neomycin, in the absence of calcium, also condensed the film but no further condensation was achieved when both neomycin and calcium were added to the subphase. Films of phosphatidylinositol and of phosphatidic acid gave essentially similar results while films of neutral phospholipids, phosphatidylcholine and phosphatidylethanolamine, did not interact significantly with either calcium or neomycin.

The behavior of a polyphosphoinositide film was markedly different (Fig. 1B). Because a stable film could not be obtained with phosphatidylinositol bisphosphate, presumably due to the hydrophilic character of the lipid, a mixed film with phosphatidylinositol phosphate was used. With this mixture normal $\pi$ vs. $A$ curves were obtained, i.e. surface tension increased with diminishing surface area similar as with other acidic phospholipids. Addition of 


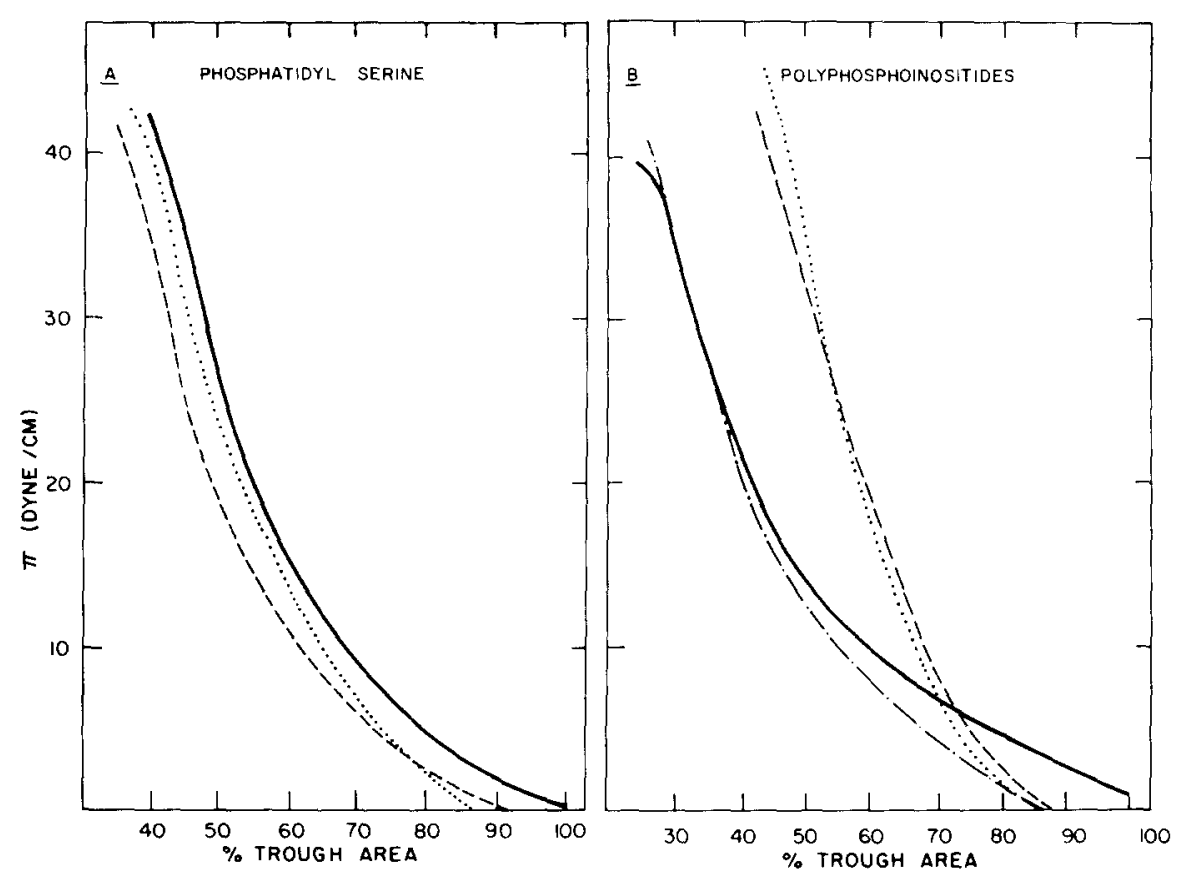

Fig. 1. Surface pressure vs. area isotherms. Films of phosphatidylserine and of polyphosphoinositides were analyzed. Films were spread over subphases containing: ___.., buffer only; ....., buffer and $1 \mathrm{mM}$ $\mathrm{CaCl}_{2} ; \cdots$, , buffer and $1 \mathrm{mM}$ neomycin; $\cdots$, , buffer and $1 \mathrm{mM}$ each of $\mathrm{CaCl}_{2}$ and neomycin. In experiment (A), the curves obtained for films spread over buffer and $1 \mathrm{~m} \mathrm{M} \mathrm{CaCl} 2$ both the presence and absence of neomycin were superimposable and are represented by the dashed line (-.....).

calcium to the subphase condensed the film at low surface pressures. The lack of a calcium effect at higher surface pressures is in apparent contrast to the results of the 'Constant Area Studies' described below (Fig. 3). It should be noted that comparative $\pi$ vs. $A$ experiments may be subject to serious errors at high surface pressures. An error of $1 \%$ in the amount of material spread over the subphase can result in differences of surface pressure of more than 10 dynes $/ \mathrm{cm}$ at areas near the limiting area of the film [12].

Neomycin produced a biphasic response: it condensed the film at low pressures while it expanded the film at higher pressures. The area/molecule at the collapse of the polyphosphoinositide/neomycin film was much greater than that of the film without the drug indicating that the drug/lipid film collapses as a single entity. This contrasts with the action of other drugs such as local anesthetics which expand lipid films but which will be expelled from an areadetermining position at higher surface pressures. Thus, the behavior of the neomycin/polyphosphoinositide film demonstrates a very strong interaction between the lipid and the drug. The curve resulting from the addition of equimolar amounts of neomycin and calcium did not differ significantly from the curve with neomycin alone.

\section{Constant area studies}

While compression isotherms are valuable for determining interactions 
between a ligand and a lipid film, they do not easily permit extrapolations to events at a biological membrane. Lipids in biological membranes are tightly packed at a high surface pressure [13] and are in equilibrium with calcium in their environment. Therefore, in order to more closely mimic physiological conditions we designed experiments for examining the effects of neomycin on tightly packed phospholipid films previously exposed to calcium. The orientation of lipid molecules in monomolecular films becomes comparable to what might be expected in the biomembrane at surface pressures of about 30 dynes/ $\mathrm{cm}$ [14]. When increasing concentrations of neomycin were added to such calcium/lipid films little or no effect of the drug on the surface pressure was seen with phosphatidylserine (Fig. 2A), phosphatidylinositol (Fig. 2B) or phosphatidic acid (Fig. 2C). In each case, omission of calcium from the system resulted in films of higher surface pressure, and neomycin produced a calcium-like condensation.

Two other lipids were included in our comparison, cardiolipin (Fig. 2D) because of its higher charge, and cerebroside sulfate (Fig. $2 \mathrm{E}$ ) because of reports that it may serve as a membrane receptor [15]. These films were condensed by the addition of neomycin both in the presence and absence of calcium.

The above results were not unique to films of high pressure but were similarly seen at lower film pressures.

Because of their non-typical behavior with neomycin polyphosphoinositide

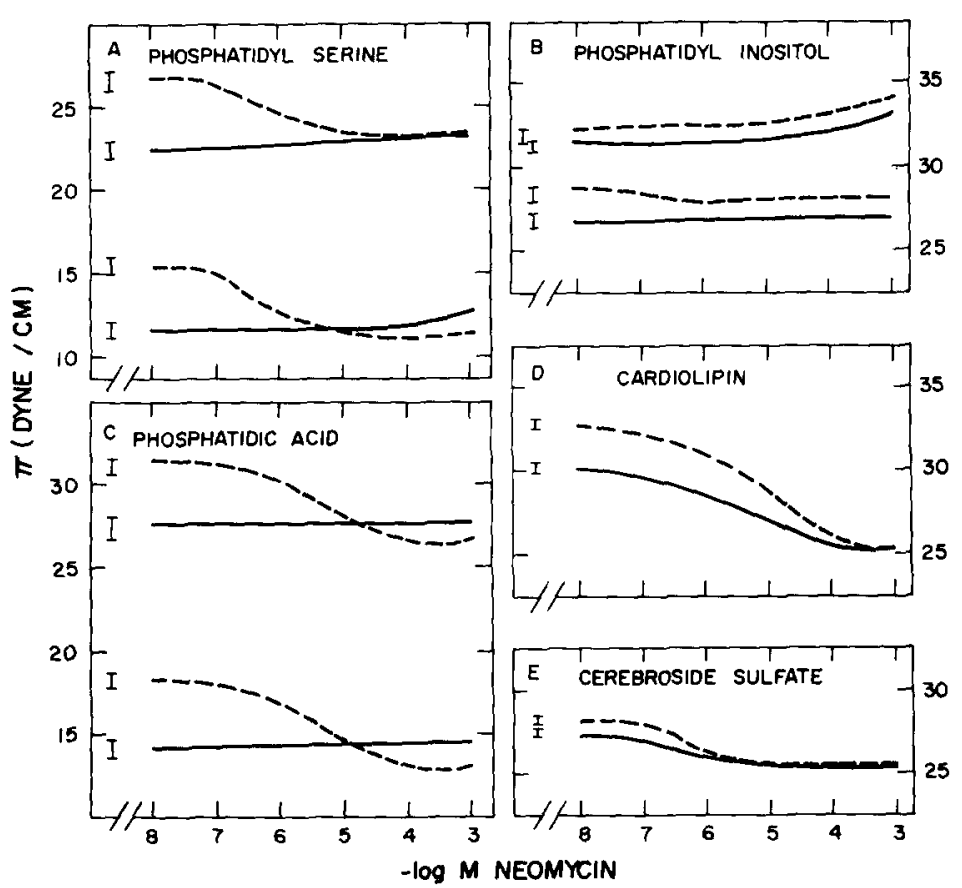

Fig. 2. Penetration curves of lipid films. Films were spread over the buffered subphase in the absence of calcium (-..) or in the presence of $1 \mathrm{mM} \mathrm{CaCl}_{2}(-$,$) . Bars at the left abscissa: range of surface$ pressure readings before the addition of neomycin. 
films are shown as a complete series of measurements at different initial surface pressures (Fig. 3A-D). For each subset of Expts. A-D the same amount of phospholipid was spread once in the absence and once in the presence of $1 \mathrm{mM}$ calcium. Calcium in the subphase considerably condensed the films and lowered their surface pressure. It was not possible to study the film described in Fig. 3D in the absence of calcium because the amount of lipid created a surface pressure which exceeded the collapse pressure of the film. Neomycin, when added in the absence of calcium caused a drop in surface pressure although in most cases much less than did calcium. This drop in surface pressure reached a minimum at $10^{-6}-10^{-5} \mathrm{M}$ neomycin and higher concentrations of the drug expanded the film again. A most dramatic effect of neomycin was seen on calcium/polyphosphoinositide films. As little as $1 \mu \mathrm{M}$ neomycin increased the surface pressure and higher drug concentrations produced surface pressures comparable to those seen in the absence of calcium. Neomycin was even able to penetrate the film of the highest initial pressure (Fig. 3D) and to increase its surface pressure to $42 \mathrm{dynes} / \mathrm{cm}$ which corresponded to the collapse pressure of the film (see Fig. 1B).

A comparison of the calcium and neomycin actions on lipid films shows that the predominant interaction of phosphatidylserine, phosphatidylinositol and phosphatidic acid was with calcium. Cardiolipin and cerebroside sulfate apparently interacted with both calcium and neomycin, each producing a condensation of the film. Polyphosphoinositide films are dominated by neomycin which produced a large expansion. Thus polyphosphoinositides appear to be unique among the various phospholipid films with respect to both the type of interaction and the magnitude of the effect.

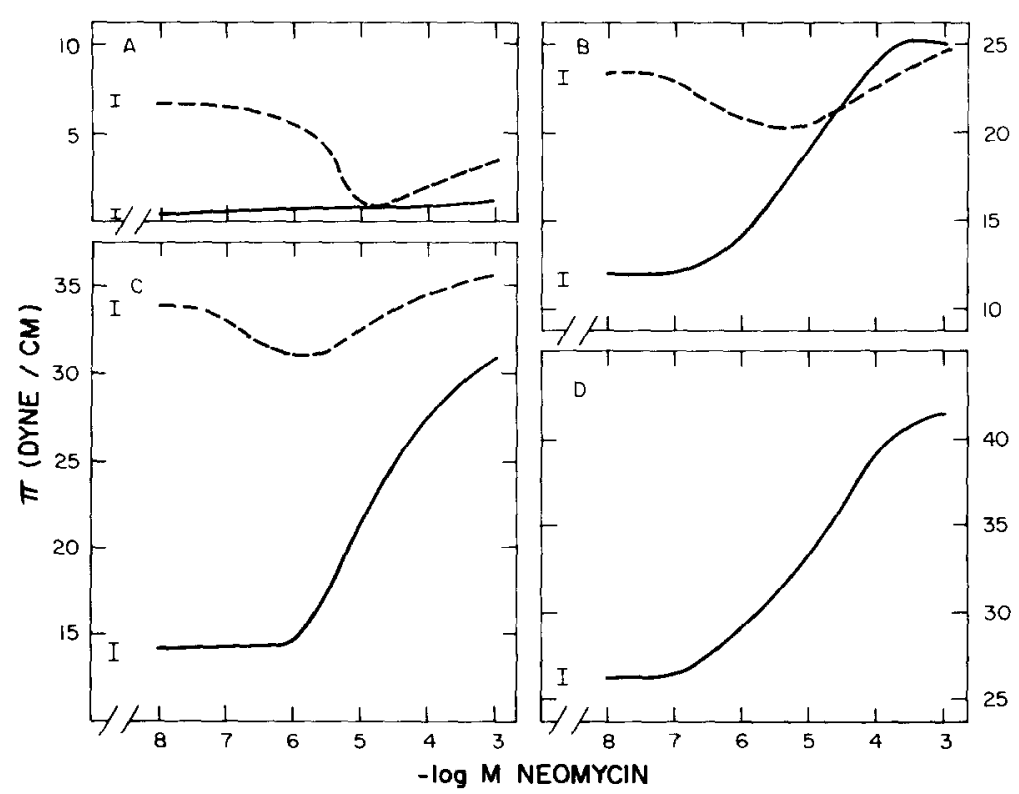

Fig. 3. Penetration curves of polyphosphoinositide films. See legend to Fig. 2. 
Interpretation of the interaction between neomycin and polyphosphoinositides

The experiments that were carried out in the absence of calcium (Fig. 3AC) provide some insight into the mechanism of the neomycin action. The appearance of the minima in the surface pressure indicates that neomycin exerts two types of effects on the lipid. First a condensation at low drug concentrations and, second, an expansion at higher concentrations. At low drug concentrations, relatively few drug molecules are expected at the interface with the lipid. The major effect at these low concentrations would be an overall reduction of the charge of the polar headgroups resulting in a reduction of the repulsive forces and a condensation of the lipid film. This nonspecific condensation effect is not unique for the polyphosphoinositide film but is observed to varying degrees with all the other anionic lipids tested. This effect can be regarded as similar to that exerted by calcium.

At a critical concentration of neomycin $\left(10^{-6}-10^{-5} \mathrm{M}\right)$ condensation of the film is maximal. Further addition of neomycin to the subphase brings more drug molecules to the interface and decreases the condensation. This decrease of condensation, seen as an increase of surface pressure, may be the result of the rather large neomycin molecule binding to polyphosphoinositides in an area-determining position. In order for a very hydrophilic penetrant like neomycin to enter into an area-determining position in the film and remain in that position up to the collapse pressure, it has to be held very rigidly at the interface. This can be accomplished by the 'penetrant' molecule attaching at two or more points on the phospholipid molecule. An examination of polyphosphoinositide and neomycin molecular models suggests that the phosphate groups of the lipids may interact primarily with two or three amino groups of the neamine moiety of neomycin, and the steric requirements for this interaction would explain why the effect is not seen with other acidic lipids. The additional amino groups on the 2- and 6-position of the neosamine moiety of the antibiotic are still available for interaction with an adjacent anionic lipid molecule. These secondary interactions would strengthen the mixed film and reaffirm the area-determining position of neomycin.

The interactions discussed thus far were in the absence of calcium and the model presented should be consistent with studies carried out in the presence of calcium. The addition of neomycin to the subphase of a calcium/polyphosphoinositide film, even at concentrations around $1 \mu \mathrm{M}$ caused an expansion. This is indicative of a very strong preference of the polyphosphoinositide film for neomycin over calcium. The expansion should be due to the fact that the phospholipid molecules are highly condensed in the presence of calcium so that the replacement of even small amounts of $\mathrm{Ca}^{2+}$ by the bulkier neomycin will produce film expansion.

\section{Conclusion}

The results obtained in the monolayer experiments are consistent with our in vivo experiments in the ear and the kidney [5-7]. The selective action of neomycin on polyphosphoinositides in those tissues can clearly be explained by a specific binding of the drug to these lipids. It seems significant for a physiological action that a concentration as low as $1 \mu \mathrm{M}$ neomycin is able to expand a 
polyphosphoinositide monolayer in the presence of $1 \mathrm{mM}$ calcium while other lipids do not show an interaction under these conditions. This evidence supports our contention that the polyphosphoinositides may serve as in vivo receptors for neomycin. Other experiments (Ref. 8; Lodhi, S., Weiner, N.D., Mechigian, I. and Schacht, J., unpublished data) indicate that the action of neomycin is shared by other toxic aminoglycosidic antibiotics which is a further indication for the possibility of a correlation between in vivo toxicity and the action on an in vitro model system.

Although polyphosphoinositides occur as trace lipids only in a number of tissues, they may serve an important role in excitable membranes. They have been reported to be concentrated in neural tissues and their monoesterified phosphate groups undergo a rapid turnover which appears to be responsive to physiological stimuli [16]. There is, however, no evidence available to ascribe these lipids a precise function. Since polyphosphoinositides avidly bind calcium, their hydrolysis and rephosphorylation may regulate membrane-bound calcium which is known to be involved in bioelectric phenomena such as nerve impulse propagation [17]. Conversely, it has been suggested that intracellular calcium levels control the rate of phosphoinositide hydrolysis [18]. In either case, binding of neomycin to polyphosphoinositides in vivo and displacement of calcium would interfere with the physiological role of these lipids explaining the toxicity of the antibiotic. In addition, from our in vivo and in vitro evidence it seems possible that neomycin may serve as a suitable tool in studies of the biochemistry and physiology of the polyphosphoinositides.

\section{Acknowledgement}

This research was supported by Grant NS-13792 and Program Project Grant NS-05785 from the National Institutes of Health.

\section{References}

1 Cuatrecasas, P. (1974) Annu. Rev. Biochem. 43, 169-214

2 Kohn, L.D., Lee, G., Grollman, E.F., Ledley, F.D., Mullin, B.R., Friedman, R.M., Meldolesi, M.F. and Aloj, S.M. (1978) in Cell Surface Carbohydrate Chemistry (Harmon, R.E., ed.), pp. 103-134, Academic Press, New York

3 Feinstein, M.B. (1964) J. Gen. Physiol. 48, 357-374

4 Teuber, M. and Miller, I.R. (1977) Biochem. Biophys. Acta 467, 280-289

5 Orsulakova, A., Stockhorst, E. and Schacht. J. (1976) J. Neurochem. 26, 285-290

6 Schibeci, A. and Schacht, J. (1977) Biochem. Pharmacol, 26, 1769-1774

7 Stockhorst, E, and Schacht, J. (1977) Acta Otolaryngol. 83, 401-409

8 Schacht, J. (1976) J. Neurochem. 27, 1119-1124

9 Lodhi, S., Weiner, N.D. and Schacht, J. (1976) Biochim. Biophys. Acta 426, $781-785$

10 Schacht, J. (1978) J. Lipid Res. 19, 1063-1067

11 Weiner, N.D., Amanat, M., Blondo, D., Caprioli, R., Dinerman, N, and Felmeister, A. (1968) J. Pharm. Sci. $57,1398-1400$

12 Felmeister, A., Amanat, M. and Weiner, N.D. (1966) J. Pharm. Pharmacol. 18, 837-838

13 Danielli, J.F. (1959) Surface Phenomena in Chemistry and Biology, pp. 246-265, Pergamon Press, New York

14 Demel, R.A., Guerts Van Kessel, W.S.M., Zwaal, R.F.A., Roelofsen, B. and Van Deenen, L.L.M. (1975) Biochim. Biophys. Acta 406, 97-107

15 Cho, J.S. and Loh, H.H. (1976) Life Sci. 18, 231-244

16 Michell, R.H. (1975) Biochim. Biophys. Acta 415, 81-147

$17 \mathrm{Kai}, \mathrm{M}$, and Hawthorne, J.N. (1969) Ann. N.Y. Acad. Sci. 165, 761-773

18 Griffin, H.D. and Hawthorne, J.N. (1978) Biochem. J. 176, 541-552 